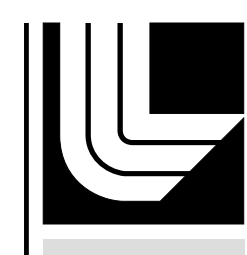

LAWRENCE LIVERMORE N A T IO N A L LABORATORY

\title{
Dispersion Analysis of the Pulseline Accelerator
}

G. J. Caporaso, R. J. Briggs, B. R. Poole, S. D. Nelson

May 12, 2005

2005 Particle Accelerator Conference (PAC 2005)

Knoxville, TN, United States

May 16, 2005 through May 20, 2005 
This document was prepared as an account of work sponsored by an agency of the United States Government. Neither the United States Government nor the University of California nor any of their employees, makes any warranty, express or implied, or assumes any legal liability or responsibility for the accuracy, completeness, or usefulness of any information, apparatus, product, or process disclosed, or represents that its use would not infringe privately owned rights. Reference herein to any specific commercial product, process, or service by trade name, trademark, manufacturer, or otherwise, does not necessarily constitute or imply its endorsement, recommendation, or favoring by the United States Government or the University of California. The views and opinions of authors expressed herein do not necessarily state or reflect those of the United States Government or the University of California, and shall not be used for advertising or product endorsement purposes. 


\title{
DISPERSION ANALYSIS OF THE PULSELINE ACCELERATOR *
}

\author{
G. J. Caporaso ${ }^{1}$, R. J. Briggs ${ }^{2}$, B. R. Poole ${ }^{1}$, S. D. Nelson ${ }^{1}$ \\ 1 Lawrence Livermore National Laboratory, Livermore, CA 94551, U.S.A. \\ 2 Science Applications International Corporation, Alamo, CA 94507, U.S.A.
}

\begin{abstract}
We analyze the sheath helix model of the pulseline accelerator [1]. We find the dispersion relation for a shielded helix with a dielectric material between the shield and the helix and compare it against the results from 3-D electromagnetic simulations. Expressions for the fields near the beam axis are obtained. A scheme to taper the properties of the helix to maintain synchronism with the accelerated ions is described. An approximate circuit model of the system that includes beam loading is derived.
\end{abstract}

\section{INTRODUCTION}

It has been recently recognized by Briggs that the wellknown slow wave structure consisting of a helix wound over an evacuated beam tube, an outer dielectric layer and an outer conductor can be used to accelerate ions [1]. When a pulse is injected into this system by impressing a voltage between the helix and outer conductor a longitudinal electric field will be generated along the helix that persists on the axis if certain conditions are satisfied. The magnitude of the electric field along the helix is roughly given by

$$
E_{z} \cong \frac{1}{v_{p}} \frac{\partial V}{\partial t}
$$

where $v_{p}$ is the phase velocity of the propagating voltage wave of amplitude $\mathrm{V}$. The helix has non-local coupling due to mutual inductance and capacitance between the windings that gives rise to dispersion. As the ions gain energy from the propagating wave their speed increases and they can lose synchronism with the accelerating field unless the properties of the helical structure are tapered in the appropriate way. There are several operating modes of the helix with respect to whether or not the wave is in synchronism with the particles. In this paper we consider only the mode in which the particles move synchronously with the wave.

\section{FIELD MODEL}

Consider the geometry shown in Figure 1. The helix has radius $a$ and the outer conductor has radius $b$. Between the helix and the outer conductor is a dielectric. The interior of the helix is in vacuum.

* This work was performed under the auspices of the U.S. Department of Energy by University of California Lawrence Livermore National Laboratory under contract No. W-7405-Eng-48.
We will use the sheath helix model to find the fields in this structure [2]. In the dielectric layer of relative permittivity $\varepsilon$ we have

$$
\left[\nabla^{2}-\frac{\varepsilon}{c^{2}} \frac{\partial^{2}}{\partial^{2}}\left(\begin{array}{l}
E_{z} \\
B_{z}
\end{array}\right)=0\right.
$$

while in the interior vacuum region we have

$$
\left\lfloor\nabla^{2}-\frac{1}{c^{2}} \frac{\partial^{2}}{\partial^{2}}\left(\begin{array}{l}
E_{z} \\
B_{z}
\end{array}\right)=0\right.
$$

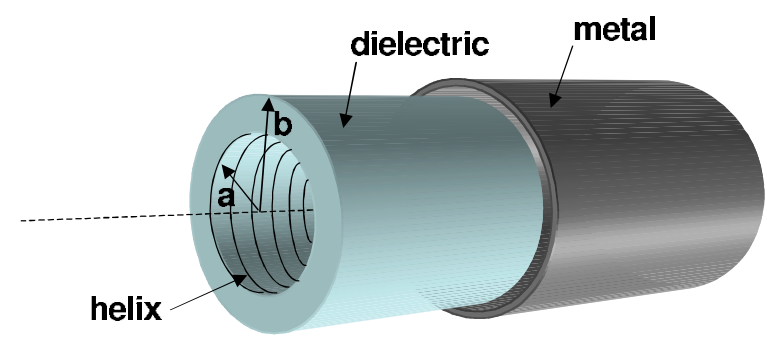

Figure 1: Schematic of helix, dielectric sleeve and outer conductor.

In the sheath helix model the actual fine structure of the helix is ignored which is a reasonable approximation for wavelengths that are long compared to the pitch or spacing of the helical windings. If we let all quantities vary as $\exp [i(k z-\omega t)]$ the field equations become

$$
\begin{aligned}
& \left\lfloor\frac{1}{r} \frac{\partial}{\partial r}\left(r \frac{\partial}{\partial r}\right)-\left(k^{2}-\frac{\varepsilon \omega^{2}}{c^{2}}\right)\right]\left(\begin{array}{c}
\tilde{E}_{z} \\
\tilde{B}_{z}
\end{array}\right)=0 \\
& \left\lfloor\frac{1}{r} \frac{\partial}{\partial r}\left(r \frac{\partial}{\partial r}\right)-\left(k^{2}-\frac{\omega^{2}}{c^{2}}\right)\left(\begin{array}{l}
\tilde{E}_{z} \\
\tilde{B}_{z}
\end{array}\right)=0\right.
\end{aligned}
$$

where equation (4) applies to the region $\mathrm{a}<\mathrm{r}<\mathrm{b}$ and equation (5) applies to the region $r<a$. The tildes indicate Fourier amplitudes. If we make the definitions $\mathrm{p}^{2}=\mathrm{k}^{2}-\varepsilon \omega^{2} / \mathrm{c}^{2}$ and $\mu^{2}=\mathrm{k}^{2}-\omega^{2} / \mathrm{c}^{2}$ then the appropriate solutions of equations (4) and (5) are 


$$
\begin{aligned}
&\left(\begin{array}{c}
\tilde{E}_{\bar{z}} \\
\tilde{B}_{z}
\end{array}\right)=\left(\begin{array}{l}
C \\
D
\end{array}\right) I_{o}(p r)+\left(\begin{array}{l}
D \\
F
\end{array}\right) K_{o}(p r) \\
&\left(\begin{array}{c}
\tilde{E}_{z} \\
\tilde{B}_{z}
\end{array}\right)=\left(\begin{array}{l}
A \\
B
\end{array}\right) I_{o}(\mu r) .
\end{aligned}
$$

By using the Maxwell curl equations all the other field components may be determined from $\mathrm{E}_{\mathrm{z}}$ and $\mathrm{B}_{\mathrm{z}}$. By imposing the condition that $\mathrm{E}_{\mathrm{z}}$ and $\mathrm{B}_{\mathrm{r}}$ vanish $\mathrm{at} \mathrm{r}=\mathrm{b}$ we find that for $\mathrm{a}<\mathrm{r}<\mathrm{b}$

$$
\begin{aligned}
& \tilde{E}_{z}=C\left[I_{o}(p r)-\frac{I_{o}(p b)}{K_{o}(p b)} K_{o}(p r)\right\rfloor \\
& B_{z}=E\left\lfloor I_{o}(p r)+\frac{I_{1}(p b)}{K_{1}(p b)} K_{o}(p r)\right\rfloor .
\end{aligned}
$$

Next, we impose the conditions the electric field tangential to the helix vanishes

$$
\tilde{E}_{z} \sin \psi+\tilde{E}_{\phi} \cos \psi=0
$$

and that the tangential magnetic field $B_{Z} \sin \psi+B_{\phi} \cos \psi$ is continuous across the helix [3].

Here the pitch angle $\psi=\cot ^{-1}(2 \pi \mathrm{a} / \mathrm{L})$ where $\mathrm{L}$ is the pitch of the helix, the distance between adjacent windings. Imposing the continuity of $\mathrm{E}_{\mathrm{z}}$ at $\mathrm{r}=\mathrm{a}$ allows the determination of all of the unknown 6 coefficients in terms of one remaining coefficient. Taking appropriate ratios of field components allows us to obtain the dispersion relation

$$
\left(\frac{\omega a}{c}\right)^{2} \cot ^{2} \psi=\frac{\frac{\mu a I_{0}(\mu a)}{I_{1}(\mu a)}+p a \frac{\frac{K_{0}(p a)}{K_{1}(p b)}+\frac{I_{0}(p a)}{I_{1}(p b)}}{\frac{K_{1}(p a)}{K_{1}(p b)}-\frac{I_{1}(p a)}{I_{1}(p b)}}}{\frac{I_{1}(\mu a)}{\mu a I_{0}(\mu a)}+\frac{\varepsilon}{p a} \frac{\frac{K_{1}(p a)}{K_{0}(p b)}+\frac{I_{1}(p a)}{I_{0}(p b)}}{\frac{K_{0}(p a)}{K_{0}(p b)}-\frac{I_{0}(p a)}{I_{0}(p b)}}}
$$

which agrees with the result obtained by Anicin [4]. A plot of $w a / c$ vs. ka is shown in Figure 2.

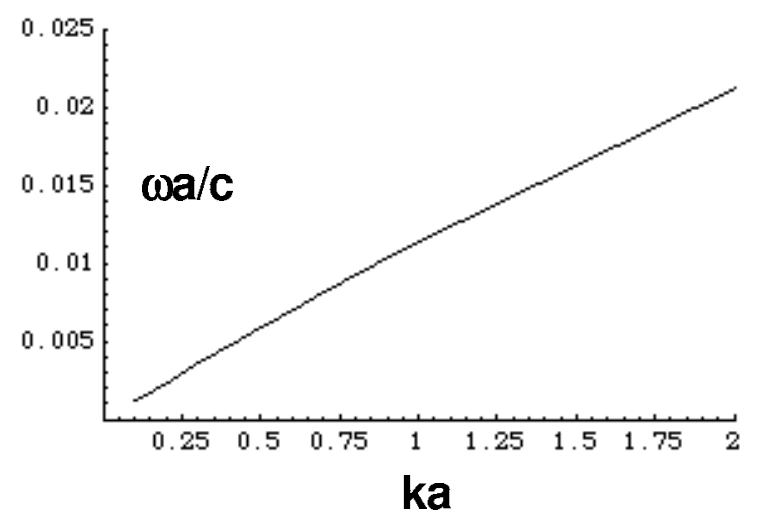

Figure 2: Plot of $\omega \mathrm{a} / \mathrm{c}$ vs. ka for the case $\varepsilon=1, \psi=.01$ and $\mathrm{b} / \mathrm{a}=1.5$.

Results of a 3-D finite difference time domain electromagnetic code XFDTD show good agreement with equation (11) [5].

Taking the limit of equation (11) as ka approaches zero yields the phase velocity $\omega / \mathrm{k}$ as

$$
v_{p}=\frac{c}{\sqrt{\varepsilon}} \sqrt{\left(1-\frac{a^{2}}{b^{2}}\right) \cot ^{2} \psi+2 \ln (b / a)} .
$$

From equation (7) and the definitions of $\mu$ and $v_{p}$ we find that

$$
\tilde{E}_{z}=\tilde{E}_{\text {wall }} \frac{I_{\iota}\left(\frac{\omega r}{\mathcal{W}_{p}}\right)}{I_{\nu}\left(\frac{\omega a}{\mathcal{W}_{p}}\right)}
$$

where

$$
\gamma=\frac{1}{\sqrt{1-\frac{v_{p}^{2}}{c^{2}}}} .
$$

From equation (13) we can see that the on-axis gradient is preserved provided that

$$
\frac{\omega a}{w_{p}}<1
$$

By relating the current in the helix to the fields the all the field components can be determined. We need to calculate the jump in $\mathrm{Bz}$ and $\mathrm{B} \phi$ across the helix

$$
B_{z>}-B_{z<}=\frac{2 I_{\phi}}{c a}
$$




$$
B_{\phi>}-B_{\phi<}=\frac{2 I_{\bar{z}}}{c a} .
$$

From the sheath helix model we have the boundary condition [2]

$$
I_{\phi} \sin \phi-I_{z} \cos \phi=0 .
$$

From the field solution we have

$$
\tilde{E}_{r}=-\frac{k}{p^{2}} \frac{\partial \tilde{E}_{z}}{\partial r}
$$

which can be related to the voltage across the line as

$$
\tilde{V}=\frac{i k \tilde{E}_{z}}{p^{2}}=\frac{i k A I_{o}(\mu a)}{p^{2}} .
$$

In the low frequency limit A can be related to the current as

$$
A=-\frac{i \omega a}{c} \frac{\tilde{I}_{z}}{c a} \frac{\cot \psi}{\tan \psi}\left(1-\frac{a^{2}}{b^{2}}\right) .
$$

Using equations (20) and (21) to relate the voltage to the current yields the characteristic impedance (in the low frequency limit) as

$$
Z=\frac{Z_{o}}{4 \pi \sqrt{\varepsilon}} \cot \psi \sqrt{\left(1-\frac{a^{2}}{b^{2}}\right) 2 \ln \frac{b}{a}} .
$$

\section{TAPERED LINE FOR SYNCHRONOUS OPERATION}

As the ions accelerate in the field of the traveling wave they will accelerate and lose synchronism with the pulse. In order to maintain synchronism the wave speed must remain equal to the ion speed

$$
v_{p}=\frac{1}{\sqrt{L C}}=v_{i} .
$$

In order that the gradient remain constant we must have

$$
E_{z}=\frac{1}{v_{p}} \frac{\partial V}{\partial t} \Rightarrow \frac{V}{v_{p}}=\text { constan } t .
$$

As we vary the parameters of the line the impedance will change. The voltage on the line will scale as the square root of the impedance. Thus we must have

$$
\frac{(L / C)^{1 / 4}}{v_{p}}=\text { constan } t .
$$

Combining equations (23) and (25) gives the tapering

$$
L \propto \frac{1}{C^{1 / 3}} \propto v_{i}
$$

\section{BEAM LOADING}

An approximate circuit model that incorporates the effects of beam loading may be written by assuming that the dispersion in the helix is small so that the propagation of electromagnetic waves is governed by the transmission line equations. We add a term proportional to the line density of the bunch

$$
\begin{aligned}
& \frac{\partial V}{\partial z}=-L \frac{\partial I}{\partial t} \\
& \frac{\partial I}{\partial z}=-C \frac{\partial N}{\partial t}+\frac{\partial \lambda_{b}}{\partial t}
\end{aligned}
$$

where $\mathrm{L}$ and $\mathrm{C}$ are respectively, the inductance and capacitance per unit length and $\lambda_{b}$ is the line charge density of the bunch.

\section{SUMMARY}

We have obtained the dispersion relation for a helix wound inside of a dielectric shell that is encased by a conducting cylinder. From the field solution in the sheath helix model we have obtained expressions for the low frequency phase velocity and characteristic impedance as well as the fields. The tapering prescription necessary to maintain synchronous acceleration with constant gradient has been derived and a simple model of beam loading has been presented.

\section{REFERENCES}

[1] R. J. Briggs, "Helical Pulseline Structures for Ion Acceleration," ROAB005, this conference.

[2] Ramo, Whinnery, Van Duzer "Fields and Waves in Communications Electronics," J. Wiley \& Sons, Inc., $3^{\text {rd }}$ ed., 1994, pgs. 476-478.

[3] R. Chatterjee, "Elements of Microwave Engineering,"J Wiley \& Sons, 1986, pg. 301.

[4] B. A. Anicin, IEE Proc. Microwave Antennas and Propagation vol. 146, No. 5, 1999.

[5] S. Nelson et. al., "Electromagnetic Simulations of Helical-Based Ion Acceleration Structures," FPAT037, this conference. 